\title{
Pd(0)-Nanoparticles Embedded in Core-Shell Nanogels as Recoverable Catalysts for the Mizoroki-Heck Reaction
}

\author{
André Pontes da Costa, ${ }^{[a]}$ Danilo Rosa Nunes, ${ }^{[a, b]}$ Mickaël Tharaud, ${ }^{[c]}$ Julie Oble, ${ }^{*[a]}$ Giovanni Poli, ${ }^{[a]}$ and \\ Jutta Rieger*[a]
}

\begin{abstract}
Core-shell nanogels are attractive stabilizers and supports for catalytically active metallic nanoparticles. Herein, we present the synthesis and the characterization of a nanostructured well-defined core-shell nanogel with the ability to stabilize palladium(0) nanoparticles in its core. This hybrid nanogel displays a remarkable stability in both solid state and in solution. This feature allowed its successful application as catalyst for the Mizoroki-Heck reaction between $n$-butyl acrylate and a series of bromo- and iodo-arenes. The yields spanned from good to excellent, and catalyst recycling could be achieved up to three times without significant activity loss. Three-phase tests indicated that the hybrid nanogel acts as a $\operatorname{Pd}(0)$ nano-reservoir. The catalysis proceeds in a quasi-homogenous fashion, with part of the catalytic activity occurring outside the nanogel explaining the observed limited recyclability.
\end{abstract}

\section{Introduction}

Palladium nanoparticles (NPs) are ubiquitous catalysts in C-C coupling reactions, including when the pre-catalyst is a soluble palladium complex. ${ }^{[1]}$ In the past decades, a special interest has been directed to understand the mode of formation of these nanoparticles during a reaction, and their catalytic mode of action. Furthermore, some efforts have been undertaken to stabilize these NPs in the reaction medium, in order to avoid the generation of inactive palladium black. The most common approach to perform C-C coupling reactions without added dative ligands makes use of tetraalkylammonium salts as stabilizers of in situ generated NPs, known as "Jeffery's ligandless conditions". ${ }^{[2]}$ The use of very low amounts of palladium catalyst without stabilizers, frequently referred to as "homeopathic palladium loading", is also a valuable alternative to molecular catalysts, since no palladium black is formed at very low catalyst concentrations. ${ }^{[3]}$

\footnotetext{
[a] Dr. A. P. da Costa, D. R. Nunes, Dr. J. Oble, Prof. G. Poli, Dr. J. Rieger

Sorbonne Universités, UPMC Univ Paris 06, CNRS, Institut Parisien de Chimie Moléculaire (IPCM), UMR 8232, 4 place Jussieu, 75005 Paris, France

E-mail: julie.oble@upmc.fr, jutta.rieger@upmc.fr

[b] D. R. Nunes

The Research Institute for Medicines (iMed. ULisboa), Faculty of Pharmacy, University of Lisbon, Av. Prof. Gama Pinto, 1649-003 Lisboa, Portugal

[c] M. Tharaud

Institut de Physique du Globe de Paris (IPGP), Sorbonne Paris Cité, Univ Paris Diderot, UMR 7154 CNRS, 1 rue Jussieu, 75005 Paris, France
}

Supporting information for this article is given via a link at the end of the document.
Immobilization of metal NPs in solid supports such as silica or carbon aerogels allowed obtaining recoverable catalysts for cross-coupling reactions, ${ }^{[4]}$ including the Mizoroki-Heck reaction. ${ }^{[5]}$ Polymers have also been used to stabilize NPs and help catalyst recycling. ${ }^{[6]}$ In recent years, soluble polymers, i.e. linear polymers, or more sophisticated structures, such as dendrimers and hyperbranched polymers have attracted attention as catalyst matrix or stabilizers, mainly due to their easy manipulation in the solid state, allowing catalyst recovery, as well as the quasi-homogenous behavior of the catalysis. ${ }^{[7]}$ However, only few examples addressed the stabilization of $\mathrm{Pd}(0) \quad \mathrm{NPs}$ by soluble polymers behaving as quasihomogeneous supports for $\mathrm{C}-\mathrm{C}$ coupling reactions. In this respect, Biffis et al. have reported the generation of palladium nanoparticles stabilized by polyacrylamide-based swellable polymeric 3D architectures, and proved their efficiency as catalysts in both Mizoroki-Heck as well as Suzuki coupling reactions. ${ }^{[8]}$

More recently, nanostructured polymeric architectures have also been used as metal supports and stabilizers for applications in catalysis. The first examples have involved the postfunctionalization of polymer NPs, usually polystyrene solid cores surrounded by a soluble cross-linked polymeric shell, able to stabilize metallic NPs. ${ }^{[9]}$ The advent and development of controlled polymerization techniques provided an easy access to a wide range of well-defined nanostructured polymers such as star-polymers, cross-linked micelles or core-shell nanogels. ${ }^{[10]}$ These new polymeric architectures, being rationally designable and able to exhibit responsive behavior, are usually classified as smart nanomaterials. ${ }^{[11]}$ However, their use as catalyst supports started to grow only recently. Thus, well-defined core-shell structures incorporating late transition metals have been synthesized and proved to be active catalysts in industrially relevant transformations such as the polymerization of methyl methacrylate $(\mathrm{Ru})^{[12]}$ and olefin hydroformylation under biphasic conditions (Rh). ${ }^{[13]}$ Nevertheless, the use of nanostructured soluble polymers as supports for quasi-homogeneous catalysis is still in its infancy and further exploration of this domain is a hot topic, expected to lead to the discovery of innovative catalytic systems.

Among the polymeric core-shell architectures, core-shell nanogels, i.e. nanometric globular-shaped polymers structured in a swollen core and surrounded by a stabilizing thin polymer shell, have recently attracted increasing interest. Thanks to the use of heterogeneous polymerization processes, combined with controlled polymerization techniques, their synthesis is now mastered. ${ }^{[14,15 b]}$

In the last years, we have developed an easy synthetic route to tailor-made core-shell nanogels by using reversible additionfragmentation chain-transfer (RAFT)-mediated aqueous 
dispersion polymerization of acrylates or acrylamides. ${ }^{[15]}$ Based on this former work, we describe here the synthesis of core-shell nanogels rationally designed to stabilize $\operatorname{Pd}(0) \mathrm{NPs}$ through its poly $(N, N$-diethylacrylamide-co- $N, N$-dimethylaminoethyl acrylate) core, and the use of this hybrid nanogel as catalyst in the Mizoroki-Heck reaction, along with its recycling. Moreover, three-phase tests were performed in order to establish the homogenous/heterogeneous character of this hybrid catalyst.

\section{Results and Discussion}

\section{Hybrid nanogel synthesis and characterization}

The core-shell nanogels were prepared according to a previously established procedure using RAFT radical polymerization in aqueous dispersion. ${ }^{[15]}$ This heterogeneous process allows the synthesis of nanometric monodisperse coreshell particles in which the core can be easily crosslinked through the use of small amounts of a bifunctional monomer, without the formation of large ill-defined gel structures (Scheme 1)..$^{14 b]}$

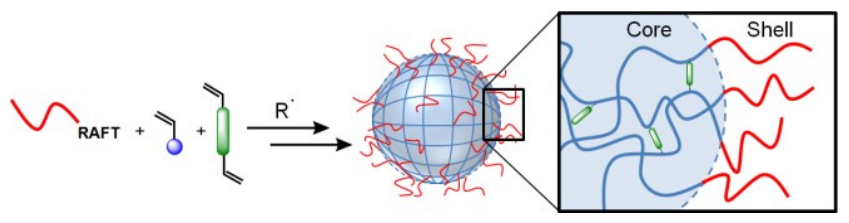

Scheme 1. Approach to core-shell nanogel architecture.

$\operatorname{Poly}(\mathrm{N}, \mathrm{N}$-dimethylacrylamide) was selected as stabilizing shell constituent for its good solubility in a large range of solvents. ${ }^{[15 a}$, ${ }^{15 c, 16]}$ Poly $(N, N$-diethylacrylamide) (PDEAAm), being also soluble in a large range of solvents, except in warm water, appeared to be the ideal candidate for the preparation of the core matrix via an aqueous heterogeneous polymerization process at moderate temperature. ${ }^{[15 a, 15 b, 16]}$ In order to provide amines required for $\mathrm{Pd}$ coordination, $\mathrm{N}, \mathrm{N}$-dimethylaminoethyl acrylate (DMAEA) was introduced in the core. In this way, the nanogel NG was obtained in water at $70^{\circ} \mathrm{C}$ by RAFT dispersion polymerization, as depicted in Scheme 2 DEAAm (85 mol\%), DMAEA (10 mol\%) and $\mathrm{N}, \mathrm{N}$ methylenebis(acrylamide) (MBA, $5 \mathrm{~mol} \%$ ) as cross-linker, in the presence of the stabilizing macromolecular RAFT agent based on poly $(N, N$-dimethylacrylamide $)$ - PDMAAm-macroRAFT, $M_{\mathrm{n}}{ }^{\text {LS }}$ $=14.2 \mathrm{~kg} \mathrm{~mol}^{-1}$ and $D=1.3$. The polymerization occurred smoothly and the reaction medium became cloudy after about 15 min, a typical signal of particle nucleation initiation in dispersion polymerization systems. After $1 \mathrm{~h}$, the monomer conversion was complete and a stable colloidal aqueous dispersion was formed. For comparison, linear PDMAAm-block$P(D E A A m-c o-D M A E A)$ chains were prepared by the same synthetic protocol but in the absence of cross-linker, see Supporting Information Table S2 and S3.

Size exclusion chromatography (SEC), dynamic light scattering (DLS) and transmission electron microscopy (TEM) assessed the formation of well-defined nanogels (NG).

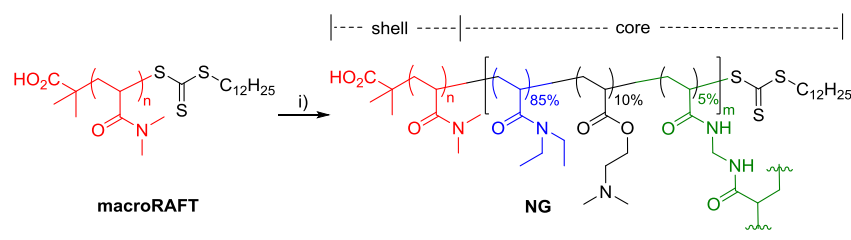

Scheme 2. Synthesis of the core-shell nanogel, PDMAAm-block-P(DEAAmco-DMAEA-co-MBA): NG. Reaction conditions: i) DEAAm 85 mol\%, DMAEA $10 \mathrm{~mol} \%$, MBA $5 \mathrm{~mol} \%, \mathrm{~V}-50, \mathrm{H}_{2} \mathrm{O}, 70^{\circ} \mathrm{C}, 1 \mathrm{~h} . \mathrm{Mn}_{\mathrm{n}}(\mathrm{PDMAAm}-\mathrm{macroRAFT})^{\mathrm{LS}}=$ $14.2 \mathrm{~kg} \mathrm{~mol}^{-1}, \oplus=1.3$.

The size exclusion chromatograms (Figure 1) evidence the complete extension of the stabilizing PDMAAm chains with the formation of high molar mass monodisperse NG, demonstrating the great efficiency of the transfer reaction. The number-average molar mass of NG was determined to be $M_{\mathrm{n}}{ }^{\mathrm{LS}}=13,400 \mathrm{~kg} \mathrm{~mol}^{-1}$ with low dispersity $(\theta=1.8)$, obtained by coupling the SEC to a static light scattering detector combined with a differential refractive index detector. Based on the observed numberaverage molar mass of $\mathbf{N G}$ and the number-average molar mass of linear chains $\left(M_{\mathrm{n}}^{\mathrm{LS}}=55.5 \mathrm{~kg} \mathrm{~mol}^{-1}, D=1.4\right)$, Figure 1 , we could estimate the number of polymer chains $\left(N_{\text {agg }}\right)$ in one NG particle to be 240 , which is in good agreement with the results obtained in the synthesis of other nanogels by a similar strategy. ${ }^{[15 c]}$ The reported reactivity ratios of DMAEA and DMAAm $\left(r_{D M A E A / D M A A m}=0.29, r_{D M A A m / D M A E A}=1.46\right),{ }^{[17]}$ reveal that DMAEA is less reactive than DMAAm, and therefore inserted preferentially at the end of the polymerization. Considering that the nanogel grows from the shell (macroRAFT) towards the core, a larger concentration of the DMAEA units deeply embedded in the core of the nanogels is expected.

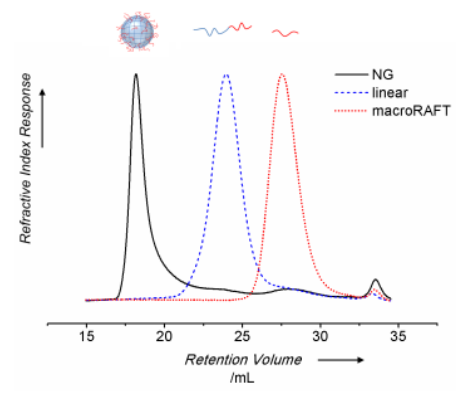

Figure 1. Size exclusion chromatography in DMF ( $\mathrm{LiBr}, 1$ wt\%) of: a) the PDMAAm-macroRAFT (dotted red line), b) PDMAAm-block-P(DEAAm-coDMAEA) linear polymer (dashed blue line), and c) PDMAAm-block-P(DEAAmco-DMAEA-Co-MBA): NG (solid black line).

Transmission electron microscopy (TEM) images of the dried, collapsed nanogel showed spherical particles, below $30 \mathrm{~nm}$ in diameter (Figure 2A). The size of the nanogels in their swollen state was then determined by DLS at $20^{\circ} \mathrm{C}$ (Figure 2B). Their z-average hydrodynamic diameter was $67 \mathrm{~nm}$ in water, and 82 $\mathrm{nm}$ in $\mathrm{N}, \mathrm{N}$-dimethylacetamide (DMAc). The observed difference in size for the dried and the swollen NG unambiguously confirms the low degree of crosslinking of the polymeric structures, 
possibly allowing the easy diffusion of small molecules, such as substrates/reactants, inside these highly swollen structures.
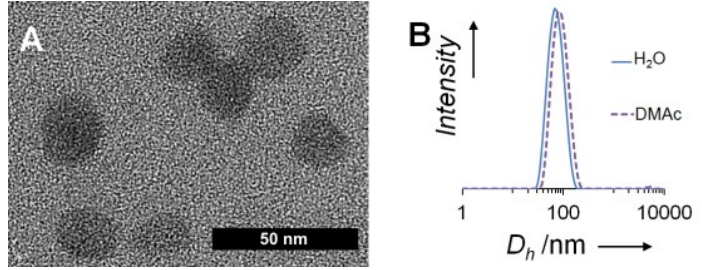

Figure 2. Size and morphology of core-shell nanogel. (A) TEM image of a dried aqueous NG solution (0.01 wt\%); (B) hydrodynamic diameter distribution obtained by DLS at $20^{\circ} \mathrm{C}$ of NG solutions $(0.1 \mathrm{wt} \%)$ in water (solid line) and DMAc (dashed line).

Embedding of $\mathrm{Pd}(0) \mathrm{NPs}$ in NG was obtained through addition of a $\mathrm{Pd}(\mathrm{II})$ salt, which is expected to be coordinated by the nitrogen functions of DMAEA units, followed by metal reduction by ethanol (Scheme 3); a method that has been already reported for this task. ${ }^{[8 b]}$ Accordingly, a solution of NG and palladium acetate in dichloromethane was initially stirred at room temperature for $24 \mathrm{~h}$ under argon atmosphere, which allowed diffusion of the metal salt into the NG. The color of the resulting solution turned from orange to dark red, indicating that coordination of the palladium atoms by the amino groups (from DMAEA) in the NG core occurred successfully. After isolation by precipitation, the $\mathrm{Pd}(\mathrm{II})$-containing $\mathrm{NGs}$ were redissolved in dichloromethane/ethanol mixture $(5: 1)$ and the solution was heated to $40^{\circ} \mathrm{C}$ for $24 \mathrm{~h}$. The solution turned brown, revealing the reduction of the metal from (II) to (0) oxidation state and formation of the NPs. ${ }^{[18]}$ After evaporation to dryness, the solid was purified by dialysis in water and lyophilized, affording a brown powder.

The integrity of the NG structure after reaction and the formation of $\mathrm{Pd}$ particles were verified by DLS after dissolution in water. No large aggregates were detected, and the PdNP@NG showed z-average hydrodynamic diameter of $104 \mathrm{~nm}$ at $20{ }^{\circ} \mathrm{C}$ (Supporting Information, Figure S4), i.e. slightly higher average diameters than the empty nanogels, which is consistent with the incorporation of $\mathrm{Pd}$ nanoparticles. TEM was used to determine the size of Pd-NPs (Figure $3 \mathrm{~A}$ ). Very small Pd particles were formed with an average diameter of $D_{\mathrm{n}}=2.9 \pm 0.6 \mathrm{~nm}$. No bigger NPs or aggregates were observed.
The hybrid NG (PdNP@NG) was further characterized by thermogravimetric analysis (TGA), X-ray photoelectron spectroscopy (XPS), and inductively coupled plasma mass spectroscopy (ICP-MS), in order to determine the percentage and oxidation state of the Pd species. The loading of palladium introduced was determined to be $1.4 \mathrm{wt} \%$ by TGA (from the weight difference between the empty NG and the hybrid NG PdNP@NG at $650^{\circ} \mathrm{C}$, see Supporting Information, Figure S3). This value was confirmed by ICP-MS (1 wt\%) and XPS (1.3 $w t \%)$ (via integration of all peaks). This value is only slightly below the maximum theoretic loading ( $1.6 \mathrm{wt} \%$ based on the amount of palladium introduced in the reaction), indicating that only few Pd atoms were lost during the PdNP@NG preparation, i.e. that most of the $\mathrm{Pd}$ was successfully supported in the NG. XPS (Figure $3 \mathrm{~B}$ ) revealed that the majority of the Pd atoms were successfully reduced to $\mathrm{Pd}(0)$, with only a few atoms in a higher oxidation state, possibly corresponding to surface atoms of the $\mathrm{Pd}(0)$ nanoparticles which are stabilized by polymeric chains of nanogel. According to the literature, ${ }^{[19]}$ we assume that the nanoparticles are stabilized by the amine function of DMAEA as well as by the amide function of DEAAm units in the nanogel core. As to the catalyst loading, we assume a $1.4 \mathrm{wt} \%$ value, as from the TGA analysis. Indeed, this technique, in contrast to ICP-MS analysis, does not involve sample digestion, and, in contrast to XPS analysis, is not surface-sensitive. In any case, as this analysis gave the highest experimental value amongst the techniques applied, assumption of this value as the most reliable one, would, in the worst case scenario, make us underestimate the amount of palladium actually present in the catalytic reactions.
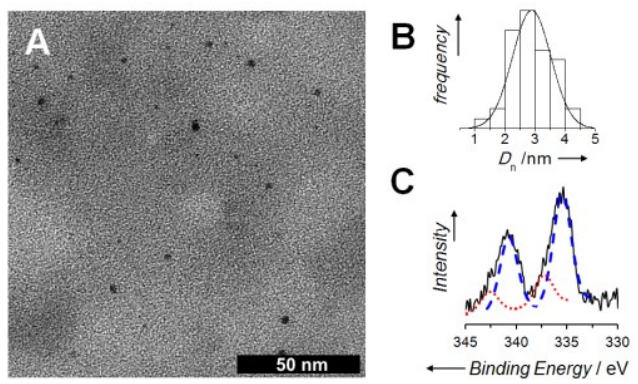

Figure 3. Size and oxidation state of palladium NPs: (A) TEM image, (B) Size distribution of the Pd NPs, and (C) XPS Pd 3d spectrum of the hybrid PdNP@NG (solid line) and its deconvolution into $\mathrm{Pd}(0)$ (dashed blue lined) and $\mathrm{Pd}(\mathrm{II})$ (dotted red line), full spectrum and assignment in the Supporting Information (Figure S5, Table S5 and S6).
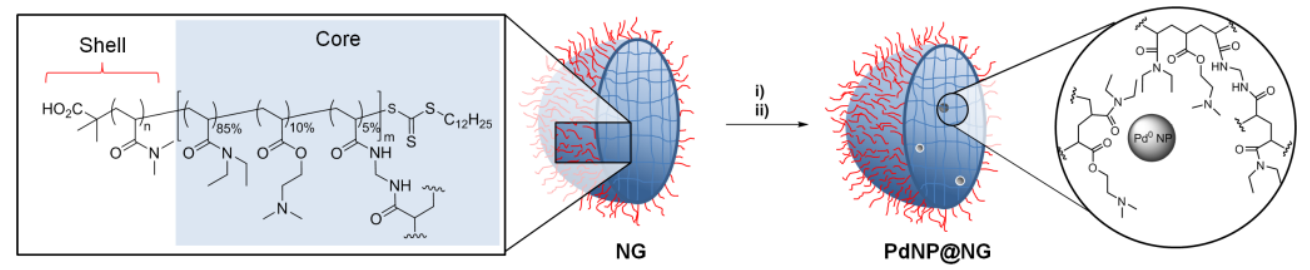

Scheme 3. Embedding of Pd(0) NPs in core-shell NG (PdNP@NG). Reaction conditions: i) Pd(OAc)2, DCM, rt, 24h. ii) EtOH, DCM, 40 $\mathrm{C}, 24 \mathrm{~h}$. 


\section{Application of PdNP@NG as catalyst in the Mizoroki-Heck reaction}

To evaluate the potential of such a novel nanostructured system as catalyst in the frame of homogeneous supported catalysis, we selected the C-C Mizoroki-Heck coupling between 4-bromo-acetophenone and $n$-butylacrylate as a benchmark reaction (Scheme 4). ${ }^{[3 b]}$

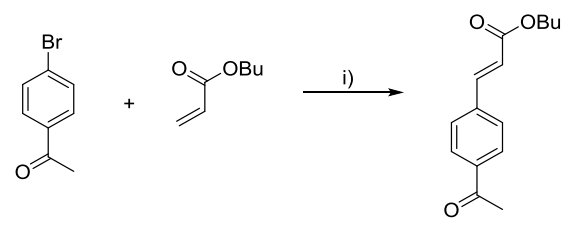

Scheme 4. The Mizoroki-Heck reaction between 4-bromo-acetophenone and $n$-butyl acrylate. Reaction conditions: i) 4-bromoacetophenone $(0.25 \mathrm{mmol})$, $n$-butyl acrylate $(0.425 \mathrm{mmol}), \mathrm{NaOAc}(0.3 \mathrm{mmol}), 0.1 \mathrm{~mol} \% \mathrm{Pd}$ (either PdNP@NG or $\left.\mathrm{Pd}(\mathrm{OAc})_{2}\right)$ and DMAc $(0.60 \mathrm{~mL})$, under argon atmosphere, $120^{\circ} \mathrm{C}$

The evolution of the reaction was investigated first (Figure 4), (round dots representing spectroscopic NMR yields of the cinnamate product). Full conversion was reached in about $2 \mathrm{~h}$ of reaction in DMAc at $120^{\circ} \mathrm{C}$. The same reaction was next repeated using a "homeopathic palladium loading"[3b] as catalyst instead of PdNP@NG. The reaction kinetics with this catalytic system was comparable to that using PdNP@NG (Figure 4, hollow squares). It thus appears that the complex structure of the core-shell NGs does not affect the catalytic performance of the palladium NPs when comparing to non-supported catalytic systems.

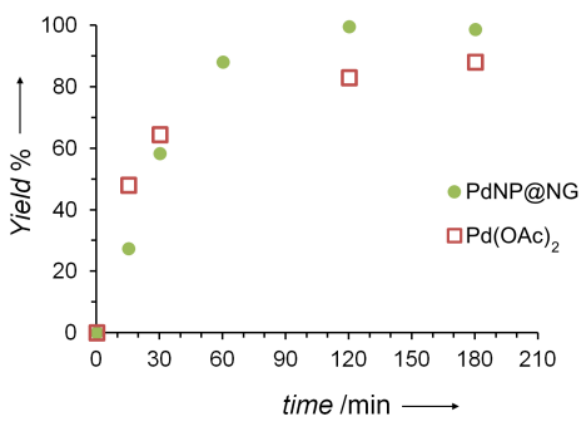

Figure 4. Kinetics of the reaction of 4-bromo-acetophenone with $n$-butyl acrylate using PdNP@NG catalyst $(\bullet)$ versus $\mathrm{Pd}(\mathrm{OAc})_{2}$ (homeopathic, $\square$ ). Reaction conditions: 4-bromoacetophenone $(0.25 \mathrm{mmol}), n$-butyl acrylate $(0.425 \mathrm{mmol}), \mathrm{NaOAc}(0.3 \mathrm{mmol}), 0.1 \mathrm{~mol} \% \mathrm{Pd}$ and DMAc $(0.60 \mathrm{~mL})$, under argon atmosphere, $120^{\circ} \mathrm{C}$. Spectroscopic yields determined by ${ }^{1} \mathrm{H}$ NMR.

These encouraging results prompted us to further explore the scope of the Mizoroki-Heck reaction catalyzed by this hybrid PdNP@NG. In this respect, the reactivity of several bromo- and iodo-arenes with $n$-butylacrylate was tested. All the reactions were conducted in the same conditions as the benchmark reaction, using 4-bromo-acetophenone (Scheme 4), and the products were isolated by flash chromatography and characterized by ${ }^{1} \mathrm{H}$ and ${ }^{13} \mathrm{C}$ NMR. The results are summarized in Table 1. The hybrid PdNP@NG was able to catalyze the coupling of $n$-butyl acrylate with bromo-arenes activated by an electron-withdrawing group in para position (entries 1 to 4). However, no reaction was observed when more electron-rich halo-arenes were used as substrates, such as chloro-benzene, bromo-benzene and 4-methoxy-bromo-benzene (entries 5, 6 and 9). In contrast, when using more reactive iodo-arenes as starting materials, the electronic nature of the substituent (hydrogen, electron-withdrawing or electron-donating group) in para or meta position did not influence the reaction yields (entries 10 to 17). Finally, low yields were obtained with bromoas well as iodo-arenes ortho-substituted with carbonyl groups (entries 7, 20 and 21), whereas a trifluoromethyl (entry 8), a methoxy (entry 18), or a methyl group (entry 19) in ortho position, allowed almost quantitative yield (>96\%). We attribute the lack of reactivity associated to the ortho-carbonyl substituted substrates to the coordinating nature of the functional group rather than to sterical hindrance. ${ }^{[20]}$ Indeed, a coordinating group in the ortho position, typically a carbonyl group, might hamper either the olefin coordination step or the formation of the cis isomer that could lead to the carbopalladation step, thus lowering the yield.

Table 1. Application of the hybrid PdNP@NG in the Mizoroki-Heck reaction ${ }^{[a, b]}$

\begin{tabular}{|c|c|c|c|}
\hline Entry & $\mathrm{X}$ & $\mathrm{R}$ & Yield $(\%)^{[b]}$ \\
\hline 1 & $\mathrm{Br}$ & $4-(\mathrm{C}=\mathrm{O}) \mathrm{CH}_{3}$ & $92^{[\mathrm{c}, \mathrm{d}]}$ \\
\hline 2 & $\mathrm{Br}$ & 4- $(\mathrm{C}=\mathrm{O}) \mathrm{H}$ & 96 \\
\hline 3 & $\mathrm{Br}$ & 4- $\mathrm{CO}_{2} \mathrm{CH}_{3}$ & 70 \\
\hline 4 & $\mathrm{Br}$ & $4-\mathrm{CF}_{3}$ & 96 \\
\hline 5 & $\mathrm{Br}$ & $\mathrm{H}$ & $\mathrm{NR}^{[\mathrm{e}]}$ \\
\hline 6 & $\mathrm{Br}$ & 4-OMe & $N R^{[e]}$ \\
\hline 7 & $\mathrm{Br}$ & 2- $\mathrm{COCH}_{3}$ & $26^{[f]}$ \\
\hline 8 & $\mathrm{Br}$ & $2-\mathrm{CF}_{3}$ & 96 \\
\hline 9 & $\mathrm{Cl}$ & $\mathrm{H}$ & $N R^{[e]}$ \\
\hline 10 & 1 & $\mathrm{H}$ & 68 \\
\hline 11 & I & $4-\mathrm{OCH}_{3}$ & 95 \\
\hline 12 & I & $4-\mathrm{CH}_{3}$ & 84 \\
\hline 13 & I & 4- $(\mathrm{C}=\mathrm{O}) \mathrm{CH}_{3}$ & $94^{[\mathrm{g}]}$ \\
\hline 14 & I & $4-\mathrm{CO}_{2} \mathrm{CH}_{3}$ & 99 \\
\hline 15 & I & $4-\mathrm{NO}_{2}$ & 69 \\
\hline 16 & I & $3-\mathrm{CH}_{3}$ & 89 \\
\hline 17 & I & 3- $\mathrm{CO}_{2} \mathrm{CH}_{2} \mathrm{CH}_{3}$ & 99 \\
\hline 18 & I & $2-\mathrm{OCH}_{3}$ & 97 \\
\hline 19 & I & $2-\mathrm{CH}_{3}$ & 98 \\
\hline 20 & I & 2- $(\mathrm{C}=\mathrm{O}) \mathrm{CH}_{3}$ & 8 \\
\hline 21 & I & $2-\mathrm{CO}_{2} \mathrm{CH}_{3}$ & 21 \\
\hline
\end{tabular}

[a] General reaction conditions: aryl halide $(0.25 \mathrm{mmol}), n$-butyl acrylate $(0.425$ $\mathrm{mmol}), \mathrm{NaOAc}(0.3 \mathrm{mmol})$, PdNP@NG $(0.1 \mathrm{~mol} \% \mathrm{Pd})$, DMAc $(0.60 \mathrm{~mL})$, $120^{\circ} \mathrm{C}, 2 \mathrm{~h}$. [b] Isolated yield after column chromatography. [c] Reaction conditions: 4-bromoacetophenone $(2.5 \mathrm{mmol}), n$-butyl acrylate $(4.25 \mathrm{mmol})$ $\mathrm{NaOAc}(3 \mathrm{mmol})$, DMAc $(6 \mathrm{~mL})$, PdNP@NG $(0.1 \mathrm{~mol} \% \mathrm{Pd}), 120^{\circ} \mathrm{C}, 2 \mathrm{~h}$. [d] TOF $=1167 \mathrm{~h}^{-1}$ at $30 \mathrm{~min}$ (58\% spectroscopic yield) [e] No reaction. [f] Average of 2 runs. [g] TOF $=1100 \mathrm{~h}^{-1}$ at $30 \mathrm{~min}$ ( $55 \%$ spectroscopic yield). 
Thus, the catalytic activity of the hybrid NG PdNP@NG in the Mizoroki-Heck reaction followed the same trend as that of the most common homeopathic ligand-free homogeneous catalysts $^{[3 b]}$ and of $\mathrm{Pd}$ NPs supported by P(DMAAm-coDMAEA), ${ }^{[8 b]}$ while showing a better performance than Pd NPs stabilized in polymeric solid supports, ${ }^{[21]}$ although this last example represents a different class of hybrid material. ${ }^{[22]}$

We then turned our attention to other olefin partners in similar reaction conditions using 4-bromo-acetophenone (Scheme 5). The hybrid PdNP@NG was moderately active in the MizorokiHeck reaction between 4-bromo-acetophenone and acrylonitrile $\left(20 \%\right.$ NMR yield in $2 \mathrm{~h}$ at $\left.120^{\circ} \mathrm{C}\right)$. In contrast, the reaction between 4-bromo-acetophenone and styrene occurred smoothly to full conversion in $2 \mathrm{~h}$ and was selective towards the $\mathrm{C}-\mathrm{C}$ coupling in the $\beta$ position. This selectivity suggests the involvement of a neutral mechanism, ruling out a cationic palladium intermediate. ${ }^{[23]}$ Intriguingly, the reaction between 4bromo-acetophenone and $n$-butyl methacrylate afforded the bisarylated product ${ }^{[24]}$ as the major product in $60 \%$ yield $^{[25]}$ (Scheme 5). Although such bis-arylated products have already been reported as side products of the Mizoroki-Heck reaction with methacrylates, optimization of their formation has never been addressed. ${ }^{[26]}$ In our case, the selectivity offered by the hybrid NG catalyst PdNP@NG in forming such bis-arylated product appears of synthetic interest.

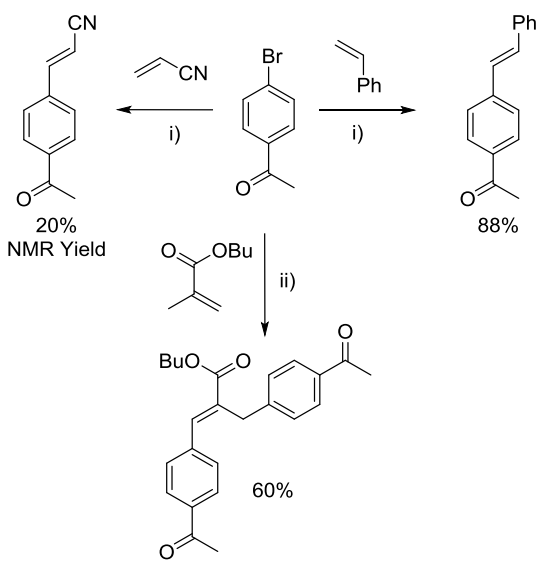

Scheme 5. Mizoroki-Heck reaction between 4-bromo-acetophenone and different olefins. Reaction conditions: i) 4-bromo-acetophenone $(0.25 \mathrm{mmol})$, alkene (0.425 mmol), NaOAc (0.3 mmol), PdNP@NG (0.1 mol\% Pd), and DMAc $(0.60 \mathrm{~mL}), 2 \mathrm{~h}, 120^{\circ} \mathrm{C}$.

\section{Hybrid nanogel recycling}

PdNP@NG recycling was investigated next. Accordingly, the model Mizoroki-Heck reaction was scaled-up 10 times with respect to the amounts used previously, so as to dispose of enough PdNP@NG for isolation purposes ( 20 mg) at the end of the cycle. The direct scale-up of the reaction afforded similar yields of isolated product as the small scale reaction. After the reaction, the hybrid NG was recovered by precipitation in diethyl ether, dried and then reused in a new cycle without any regeneration. The amount of the new starting material was maintained unchanged in the different recycling steps, independently of the catalyst mass loss during the recovery protocol (overall $10 \mathrm{wt} \%$ after 3 cycles). The results over 4 catalytic cycles are depicted in Figure 5 . The catalytic activity turned out to be steady during the first 3 cycles $(\sim 90 \%$ isolated yield), reaching an accumulated turnover number (TON) of 2880 mol of product per mol of palladium. However, in the fourth cycle, the spectroscopic NMR yield dropped to $33 \%$, indicating a clear erosion of the NG catalytic activity.

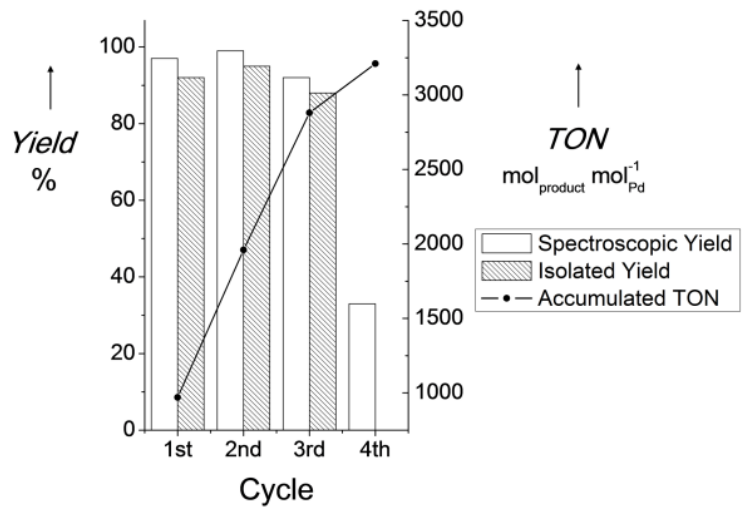

Figure 5. Spectroscopic NMR yield (solid white bar), isolated yield (diagonally striped bar) and accumulated turnover number (black dots) for the recycling of the hybrid PdNP@NG. Reaction conditions: Reaction conditions per cycle: 4bromoacetophenone $(2.5 \mathrm{mmol}), n$-butyl acrylate $(4.25 \mathrm{mmol}), \mathrm{NaOAc}$ (3 $\mathrm{mmol})$, DMAc $(6 \mathrm{~mL}), 120^{\circ} \mathrm{C}, 2 \mathrm{~h}$, PdNP@NG $(0.1 \mathrm{~mol} \% \mathrm{Pd})$ was added in the first cycle and recycled thereafter.

To better understand the drop in activity, the hybrid NG recovered after the fourth catalytic cycle was analyzed by TEM, XPS and ICP-MS. The TEM image revealed the presence of large $\mathrm{Pd}$ aggregates, reaching up to several hundreds of nanometers (Figure 6A). Moreover, XPS analyses proved that most of the palladium $(93 \%)$ recovered at this stage was oxidized to $\mathrm{Pd}(\mathrm{II})$ (Figure 6B and Table 2).
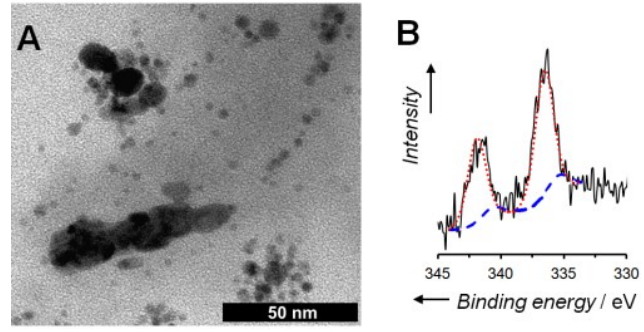

Figure 6. (A) TEM image and (B) XPS Pd 3d spectrum XPS Spectra of the hybrid PdNP@NG after 4 catalytic cycles (solid black line) and its deconvolution into $\operatorname{Pd}(0)$ (dashed blue lined) and $\mathrm{Pd}(\mathrm{II})$ (dotted red line), full spectrum in the supporting information (Figure S7, Table S8 and S9). 
Moreover, ICP-MS analysis of the isolated nanogel revealed an important loss (about $80 \mathrm{wt} \%$ ) of palladium from the polymeric nanogel support. The nanogel recovered after the fourth run contained only $0.2 \mathrm{wt} \% \mathrm{Pd}$ instead of the initial $1 \mathrm{wt} \%$. Table 2 summarizes the palladium content and oxidation state of the hybrid nanogel before and after catalysis (determined by ICP-MS and XPS). It is noteworthy to mention that the integrity of the polymeric nanogel architecture was maintained in the course of the four catalytic cycles. By DLS no significant change in the PdNPs@NG size distribution was observed (see Supporting Information Figure S6). These combined analyses point out that palladium leaches out of the NG in the course of the reaction, and that large $\mathrm{Pd}$ aggregates are formed outside the nanogels. These Pd aggregates are hardly protected - as no stabilizing polymer chain is present - and thus more prone to oxidation. The size increase (up to hundreds of nanometers) could be caused by Ostwald ripening ${ }^{[27]}$ in absence of stabilizer, and it confirms that the small nanoparticles initially formed by the reduction of $\mathrm{Pd}(\mathrm{OAc})_{2}$ with ethanol were generated in the confined NG core. It should be reminded that in the initial TEM micrographs no bigger particles or particles aggregates were observed. All in all, we attribute the activity drop to the combination of palladium loading reduction, virtual absence of $\mathrm{Pd}(0)$ species and decrease in surface area.

Table 2. Palladium content and oxidation state in the hybrid nanogel before and after catalysis.

\begin{tabular}{cccccc}
\hline \multirow{2}{*}{ Entry } & & \multicolumn{2}{c}{ Pd content (wt\%) } & \multicolumn{2}{c}{$\begin{array}{c}\text { Oxidation state } \\
(\%)^{[a]}\end{array}$} \\
& & ICP-MS & XPS $^{[\mathrm{b}]}$ & $\mathrm{Pd}^{11}$ & $\mathrm{Pd}^{0}$ \\
\hline 1 & Before catalysis & 0.95 & 1.3 & 24 & 76 \\
2 & After 4 cycles (2 h each) & 0.22 & 0.5 & 93 & 7 \\
\hline
\end{tabular}

[a] Atomic percentage from the deconvolution of the Pd 3d XPS peak. [b] Wt\% from the integration of all XPS peaks: $C$ 1s, $N$ 1s, O 1s and $P d 3 d$.

Finally, the amount of palladium leached into the organic phase during catalysis was assessed. To this end, after the first cycle and subsequent catalyst recovery by precipitation in diethyl ether, the supernatant was washed with water and evaporated to dryness, allowing to determinate the Pd contents in both the organic phase and the corresponding aqueous phase. ICP-MS revealed that the organic phase contained $77 \mathrm{ppm}$ of palladium, which, considering $1.4 \mathrm{wt} \%$ of Pd in the PdNP@NG, corresponded to about $20 \%$ of the total palladium introduced in the catalytic reaction (Table 3 ). In contrast, the aqueous phase contained only traces of the metal $(0.04 \mathrm{ppm}$ that corresponds to $2 \%$ of the total Pd introduced).

Table 3. Palladium leaching out of the nanogel.

\begin{tabular}{cccc}
\hline Entry & Phase & \multicolumn{2}{c}{ Pd content } \\
& & ppm & $\%$ total Pd \\
\hline 1 & Organic & 76.6 & 19 \\
2 & Aqueous & 0.04 & 2 \\
\hline
\end{tabular}

\section{Palladium Leaching test /three phase test}

Palladium leaching from the PdNP@NG to the organic solution $^{[28]}$ could occur at different stages: either early in the catalytic reaction, meaning that most of the $\mathrm{C}-\mathrm{C}$ coupling reaction would occur outside of the nanogel, or at the end of the catalytic reaction, after that most of the substrate is consumed or even during the work-up, i.e. during the recovery of the NG by precipitation. In order to unambiguously determine when $\mathrm{Pd}$ leaching occurs, we decided to run some test reactions. We setup a typical three-phase test ${ }^{[29]}$ using either a polymeric palladium scavenger or a polymeric substrate, which cannot diffuse to the core of the polymeric NG due to steric and entropic reasons. ${ }^{[30]}$

First, poly(4-vinylpyridine) (P4VP) was tested as a palladium scavenger. ${ }^{[31]}$ Two reactions were run in parallel in which $1 \mathrm{~mL}$ of DMAc, in the presence or absence of P4VP, was added after 30 min reaction. ${ }^{[32]}$ The kinetics is shown in Figure 7.

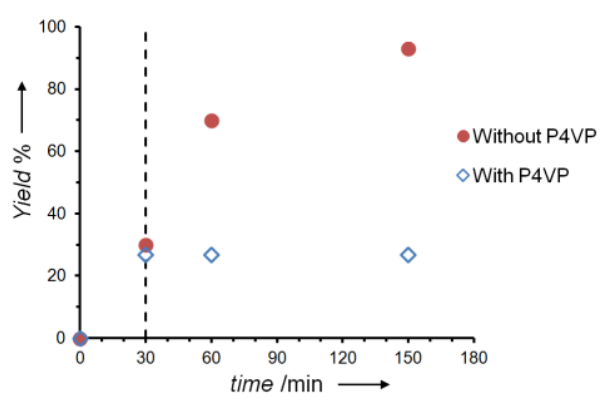

Figure 7. Effect of the addition of poly(4-vinylpyridine) $\left(M_{\mathrm{w}}=50 \mathrm{~kg} \mathrm{~mol}^{-1}\right)$ to the Mizoroki-Heck reaction catalyzed by PdNP@NG. Reaction conditions: 4 bromoacetophenone $(0.25 \mathrm{mmol}), n$-butyl acrylate $(0.425 \mathrm{mmol}), \mathrm{NaOAc}(0.3$ mmol), PdNP@NG (0.1 mol\% Pd) and DMAc (4 mL), after 30 minutes reaction $1 \mathrm{~mL}$ of DMAc is added, with or without P4VP $(50 \mathrm{mg})$. Spectroscopic yields determined by ${ }^{1} \mathrm{H}$ NMR.

The observed instantaneous inhibition of the reaction after the addition of $\mathrm{P} 4 \mathrm{VP}$, i.e. the palladium sequestrating polymer, indicates that after $30 \mathrm{~min}$ (at the latest) most of the active palladium species were outside of the nanogel.

Concerning the use of polymeric substrates, our initial approach was to use a PEGylated ester of 4-bromo-benzoic acid. However, this substrate gave no conversion, even when using soluble $\mathrm{Pd}(\mathrm{OAc})_{2}$ as catalyst. This result emphasizes the importance of a judicious choice of substrates for the threephase test, and, as already pointed out, ${ }^{[29 \mathrm{~d}-\mathrm{f}]}$ alerts that the absence of catalysis is a necessary, but not a sufficient condition to prove a purely heterogeneous catalytic behavior. We next performed our test on the commercially available poly(ethylene glycol) methacrylate $\left(M_{\mathrm{n}}=1.1 \mathrm{~kg} \mathrm{~mol}^{-1}\right)$, which is expected to react only in the second step of the catalytic cycle (the carbopalladation step). In this case, the reaction was successful, affording, after $2 \mathrm{~h}$ at $120^{\circ} \mathrm{C}, 80 \%$ yield of products with $60 \%$ selectivity toward the bis-arylated product (Scheme 6 ). 


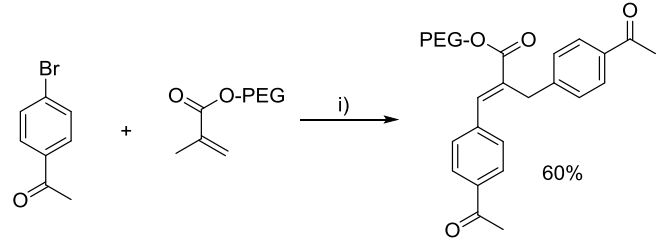

Scheme 6. Mizoroki-Heck reaction with polymeric methacrylate substrate Reaction conditions: i) 4-bromoacetophenone $(0.25 \mathrm{mmol})$, PEG-methacrylate (0.425 mmol), NaOAc (0.3 mmol), PdNp@NG (0.1 mol\% Pd) and DMAc $(0.60$ $\mathrm{mL}), 120^{\circ} \mathrm{C}, 2 \mathrm{~h}$. PEG-methacrylate $M_{\mathrm{n}}=1.1 \mathrm{~kg} \mathrm{~mol}^{-1}$.

These results clearly indicate that palladium is leaching out of the nanogel in an early stage of the reaction, and that these soluble palladium species (outside the nanogel) are responsible for most, if not all, the catalytic activity of the hybrid nanogel. We estimate the amount of palladium leached out by the end of the reaction to be about $20 \%$ of the total palladium introduced into the reaction. This figure is in agreement with the $20 \%$ of palladium found in the organic phase, by ICP-MS, and with the total amount of palladium lost in the course of 4 catalytic cycles $(80 \%)$, as shown in Tables 2 and 3, respectively. The extent of palladium leaching is in line with the observed behavior in $\mathrm{Pd}$ NPs stabilized in zeolites, or dispersed in ionic liquids during the Mizoroki-Heck reaction. ${ }^{[33]}$

This scenario is also in agreement with NG activity being of the same order of magnitude as those of soluble/homogeneous systems, but much better than Pd nanoparticles supported in polymeric solid, supports where leaching was shown to be irrelevant. $^{[21]}$

With this in mind, we propose a plausible reaction mechanism for this PdNP@NG catalyzed transformation (Scheme 7). Oxidative addition of the halo-arene to $\mathrm{Pd}(0)$ generates the corresponding $\sigma$-aryl-palladium complex $\mathbf{A}(\operatorname{ArPdX})$, expected to be soluble in the reaction media and stable enough to diffuse outside the nanogel. Subsequent alkene coordination to complex A followed by syn carbopalladation leads to complex $\mathbf{B}$, which, after syn- $\beta$-hydride elimination, affords the arylated alkene and hydridopalladium(II) complex C (HPdX). Finally, reversible reductive elimination of $\mathbf{C}$ regenerates the active $\operatorname{Pd}(0)$ complex, thereby closing the catalytic cycle. The base shifts this equilibrium toward the $\operatorname{Pd}(0)$ catalyst by scavenging the generated hydrogen halide. During catalyst turnover, the initially tiny Pd NPs become larger and larger Pd aggregates with decreasing oxidative addition power. The proposed catalyst life-cycle is in agreement with the most commonly accepted reactivity of palladium nanoparticles as catalyst in the MizorokiHeck reaction in organic solvents. ${ }^{[34]}$

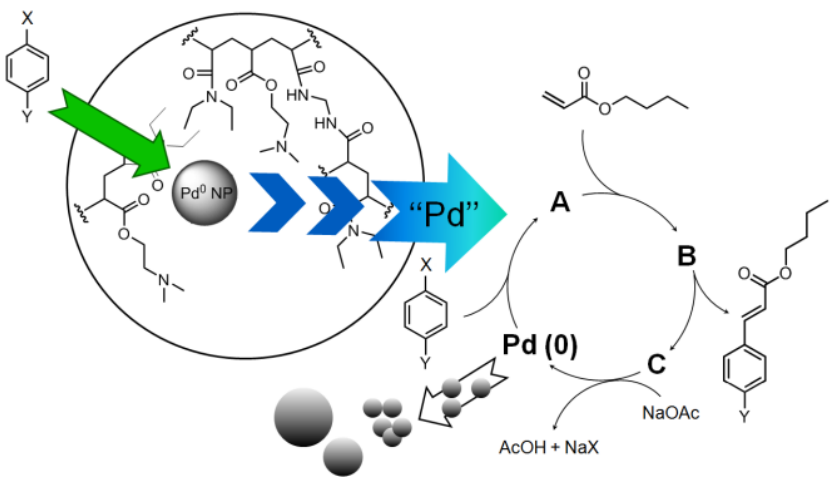

Scheme 7. Proposed catalyst life-cycle of the hybrid PdNP@NG

\section{Conclusions}

In conclusion, we have shown that RAFT dispersion polymerization can be a useful approach for the preparation of well-defined core-shell NG functionalized with amino groups in the core. The rational design of the core afforded a NG that could be readily doped with $\operatorname{Pd}(0)$ NPs. The hybrid NG could be isolated a solid powder, and the $\operatorname{Pd}(0) \mathrm{NPs}$ in its core showed remarkable stability for long periods ( $>1$ year), even in the presence of air and moisture. Therefore, the NG reported in this work is a readily available and easy to handle $\mathrm{Pd}(0) \mathrm{NP}$ reservoir.

The hybrid NG displayed good activities as catalyst in the Miroroki-Heck reaction between $n$-butyl acrylate and activated bromo-arene and iodo-arenes. Moreover, this hybrid catalyst could be recycled up to three times by precipitation, reaching an accumulated TON of 2880. However, the catalyst lost most of its activity after three cycles, which was attributed to palladium leaching out of the NG into the organic solution.

Palladium leaching seems to occur during the catalysis, probably after the oxidative addition step, which is expected to take place in the core of the NG. This leaching represents a loss of about $20 \%$ per cycle of total palladium introduced in the system.

We have thus demonstrated that core-shell NGs are promising catalyst supports, showing a quasi-homogeneous behavior while allowing catalyst recycling, although improvements in the NG design will be needed for future developments of this strategy in order to avoid palladium leaching into the organic phase during catalysis. Notably, one of the main advantages of using RAFT dispersion polymerization is the ability to fine tune the nanogel architecture. The coordinating/stabilizing nature of the core polymer and the cross-linking degree will be considered in future developments of this project. 


\section{Experimental Section}

\section{Materials}

$N, N$-Diethylacrylamide (DEAAm) ${ }^{[35]}$ and the macromolecular RAFT agent (PDMAAm-macroRAFT, $\left.M_{\mathrm{n}}^{\mathrm{LS}}=14.2 \mathrm{~kg} \cdot \mathrm{mol}^{-1}, \quad \boxminus=1.3\right)^{[16]}$ were synthesized according to published procedures. $N, N$-Dimethylacrylamide (DMAAm), N,N-diethylacrylamide (DEAAm) and $\mathrm{N}, \mathrm{N}$-dimethylaminoethyl acrylate (DMAEA) were distilled prior to polymerization. All other reagents and solvents were obtained from commercial sources and used as received.

\section{Synthesis of NG}

The PDMAAm-macroRAFT (590 mg, $41.5 \mu \mathrm{mol})$, DEAAm (1.48 g, 11.6 mmol), DMAEA (196 mg, $1.4 \mathrm{mmol})$, MBA (105 mg, $0.68 \mathrm{mmol}$ ) and 1,3,5-trioxane (123 mg, $1.4 \mathrm{mmol}$ ) were in dissolved $45 \mathrm{~mL}$ of distilled water, in a septum-sealed flask. The mixture was purged with argon in an ice-bath for $30 \mathrm{~min}$. After heating to $70^{\circ} \mathrm{C}, 1 \mathrm{~mL}(5.5 \mu \mathrm{mol})$ of an aqueous $\mathrm{V}-50$ stock solution $(5.5 \mathrm{mM})$ was added under inert atmosphere. The reaction was nearly complete after $30 \mathrm{~min}$ and it was stopped after $1 \mathrm{~h}$ by cooling in an ice bath and opening to air. Conversion was determined by the integration of olefinic protons using 1,3,5-trioxane as reference. The reaction was dialyzed against distilled water and the final solution freezedried affording the core-shell nanogel NG as a white powder $(1.5 \mathrm{~g})$.

\section{Synthesis of PdNP@NG}

A solution of NG $(1.0 \mathrm{~g})$ and $\mathrm{Pd}(\mathrm{OAc})_{2}(33 \mathrm{mg}, 0.15 \mathrm{mmol})$ in $40 \mathrm{~mL}$ of dichloromethane was purged with argon and stirred under inert atmosphere. After $24 \mathrm{~h}$ the reaction mixture was poured into $150 \mathrm{ml}$ of diethyl ether, the precipitate washed with diethyl ether $(3 \times 50 \mathrm{~mL})$ and dried under vacuum. The dark red powder was dissolved in $30 \mathrm{~mL}$ of dichloromethane and purged with argon. Then $6 \mathrm{~mL}$ of ethanol were added the reaction mixture was heated to $40^{\circ} \mathrm{C}$ under inert atmosphere. After $24 \mathrm{~h}$ the reaction mixture was poured into $150 \mathrm{ml}$ of diethyl ether and the precipitate washed with diethyl ether $(3 \times 50 \mathrm{~mL})$ and dried under vacuum. The brown solid was dissolved in $20 \mathrm{~mL}$ of distilled water and dialyzed against distilled water. The final solution freeze-dried affording the PdNP@NG as a brown powder $(0.6 \mathrm{~g})$.

\section{General Mizoroki-Heck reaction}

The arene $(0.25 \mathrm{mmol})$ and sodium acetate $(25 \mathrm{mg}, 1.2 \mathrm{eq}, 0.3 \mathrm{mmol})$ were added to a solution of alkene (1.7 eq, $0.425 \mathrm{mmol})$ in $\mathrm{N}, \mathrm{N}$ dimethylacetamide (DMAc) $(0.3 \mathrm{~mL})$. Then $0.3 \mathrm{~mL}(0.25 \mu \mathrm{mol}$ of $\mathrm{Pd}, 0.1$ mol\%) of a stock solution of PdNP@NG in DMAc $(7 \mathrm{mg} / \mathrm{mL})$ were added and the mixture purged with argon for $15 \mathrm{~min}$. The reaction was stirred at $120^{\circ} \mathrm{C}$ under inert atmosphere for $2 \mathrm{~h}$. The crude product was purified by Combi-flash chromatography in silica gel using a gradient from pentane to pentane/diethyl ether $(4: 1)$ as eluent. The fractions were evaporated to dryness and dried under vacuum.

\section{Catalyst recycling}

Catalyst recycling experiments were run using 4-bromoacetophenone and $n$-butyl acrylate as substrates following the above protocol scaled-up ten times. The catalyst was recovered after $2 \mathrm{~h}$ reaction time by pouring the reaction mixture into cold diethyl ether $(100 \mathrm{~mL})$. The recovered solid was dissolved in $10 \mathrm{~mL}$ of distilled water, dialyzed against distilled water, freeze-dried and used as recovered in the following catalytic cycle.
Full instrumental details and product characterization are given in the supporting information.

\section{Acknowledgements}

The authors would like to acknowledge Labex Michem for funding the NACRE project as well as a post-doc fellowship (A.P.C.). CNRS, UPMC and Horizon 2020 European Research Area Network, ERANet-LAC (ref. ELAC2014/BEE-0341) project CelluloseSynThech are also acknowledged for financial support. ICP-MS analyses were supported by IPGP multidisciplinary program PARI, and by Region Île-de-France SESAME Grant no. 12015908. UPMC collaborators, Omar Khaled (IPCM) for the HRMS analyses, Patricia Beaunier (LRS) and Jean-Miche Guigner (IMPMC) for TEM analyses and Christophe Calers (LRS) for XPS analysis are thanked. The authors would like to acknowledge Dr. François Stoffelbach for valuable discussions.

Keywords: Core-Shell Nanogel • RAFT-Polymerization • Palladium • Mizoroki-Heck Reaction $\bullet$ Recycling

[1] a) M. T. Reetz, J. G. de Vries, Chem. Commun. 2004, 1559-1563; b) D. Astruc, Inorg. Chem. 2007, 46, 1884-1894; c) J. Durand, E. Teuma, M. Gómez, Eur. J. Inorg. Chem. 2008, 3577-3586; d) C. Deraedt, D. Astruc, Acc. Chem. Res. 2014, 47, 494-503.

[2] a) T. Jefferey, J. Chem. Soc., Chem. Commun. 1984, 1287-1289; b) T. Jefferey, Tetrahedron 1996, 52, 10113-10130.

[3] a) M. T. Reetz, E. Westermann, R. Lohmer, G. Lohmer, Tetrahedron Lett. 1998, 39, 8449-8452. b) A. H. de Vries, J. M. Mulders, J. H. Mommers, H. J. Henderickx, J. G. de Vries, Org. Lett. 2003, 5, 32853288

[4] S. Cacchi et al., Tetrahedron 2007, 63, 2519-2523, see Supporting Information.

[5] a) S. Martínez, A. Vallribera, C. L. Cotet, M. Popovici, L. Martín, A. Roig M. Moreno-Mañas, E. Molins, New J. Chem., 2005, 29, 1342-1345; b) S. Martínez, M. Moreno-Mañas, A. Vallribera, U. Schubert, A. Roig, E. Molins, New J. Chem., 2006, 30, 1093-1097. c) L. Martín, E. Molins, A Vallribera, New J. Chem., 2016, 40, 10208-10212.

[6] a) J. Cookson, Platinum Met. Rev. 2012, 56, 83-98; b) P. TaladrizBlanco, P. Hervés, J. Pérez-Juste Top. Catal. 2013, 56, 1154-1170.

[7] a) R. Haag, S. Roller, Top. Curr. Chem. 2004, 242, 1-42; b) D. E. Bergbreiter, J. Tian, C. Hongfa, Chem. Rev. 2009, 109, 530-582; c) D. E. Bergbreiter, ACS Macro. Lett. 2014, 3, 260-265; d) Y. Lu, M. Ballauff, Prog. Polym. Sci. 2016, 59, 89-104.

[8] a) A. Biffis, J. Mol. Catal. A : Chem. 2001, 165, 303-307 ; b) A. Biffis, N. Orlandi, B. Corain, Adv. Mater. 2003, 15, 1551-1555; c) A. Biffis, E Sperotto, Langmuir 2003, 19, 9548-9550; d) L. Minati, B. Biffis, Chem. Commun. 2005, 1034-1036.

[9] Y. Lu, N. Welsch, J. Dzubiella, M. Ballauff, Prog. Colloid Polym. Sci. 2013, 140, 113-130.

[10] a) K. Matyjaszewski, J. Spanswick Mater. Today (Oxford, U. K.) 2005, 8 26-33; b) W. A. Braunecker, K. Matyjaszewski, Prog. Polym. Sci. 2007 32, 93-146.

[11] M. A. C. Stuart et. al., Nat. Mater. 2010, 9, 101-113, see Supporting Information.

[12] T. Terashima, A. Nomura, M. Ito, M. Ouchi, M. Sawamoto, Angew. Chem. Int. Ed. 2011, 50, 7892-7895.

[13] a) X. Zhang et. al., Chem. Eur. J. 2014, 20, 15505-15517, see Supporting Information; b) A. F. Cardozo et. al., J. Catal. 2015, 324, 1-8, see Supporting Information; c) S. Chen et. al., Polymer 2015, 72, 327- 
335, see Supporting Information. d) S. Chen, F. Gayet, E. Manoury, Ah Joumaa, M. Lansalot, F. D'Agosto, R. Poli, Chem. Eur. J. 2016, 22 $6302-6313$.

[14] a) J. Poly, D. J. Wilson, M. Destarac, D. Taton, Macromol. Rapid Commun. 2008, 29, 1965-1972; b) J. Rieger, Macromol. Rapid Commun. 2015, 36, 1458-1471; c) P. B. Zetterlund, S. C. Thickett, S. Perrier, E. Bourgeat-Lami, M. Lansalot, Chem. Rev. 2015, 115, 97459800.

[15] a) J. Rieger, C. Grazon, B. Charleux, D. Alaimo, C. Jérôme, J. Polym. Sci., Part A: Polym. Chem. 2009, 47, 2373-2390; b) N. Sanson, J. Rieger, Polym. Chem. 2010, 1, 965-977; c) C. Grazon, J. Rieger, N. Sanson, B. Charleux, Soft Matter 2011, 7, 3482-3490.

[16] J. Rieger, W. Zhang, F. Stoffelbach, B. Charleux, Macromolecules 2010, $43,6302-6310$.

[17] a) S. A. Pooley, B. L. Rivas, G. D. C. Pizarro, J. Chil. Chem. Soc. 2013 , 58, 1597-1602.

[18] Pictures showing the colour change in the course of the preparation of the hybrid nanogel can be found in the Supporting Information (Figure S2)

[19] a) S. V. Vasilyeva, M. A. Vorotyntsev, I. Bezverkhyy, E. Lesniewska, O. Heintz, R. Chassagnon, J. Phys. Chem. C 2008, 112, 19878-19885; b) M. Zecca, R. Fišera, G. Palma, S. Lora, M. Hronec, M. Králik, Chem. Eur. J. 2000, 6, 1980-1986

[20] M. Feuerstein, H. Doucet, M. Santelli, J. Org. Chem. 2001, 66, 59235925.

[21] L. Yu, Y. Huang, Z. Wei, Y. Ding, C. Su, Q. Xu, J. Org. Chem. 2015, 80 8677-8683.

[22] For recent examples, see the following references: a) S. Moussa, A. R. Siamaki, B. F. Gupton, M. S. El-Shall, ACS Catal. 2012, 2, 145-154; b) L. Liang, A. K. Diallo, L. Salmon, J. Ruiz, D. Astruc, Eur. J. Inorg Chem. 2012, 2950-2958; c) F. Giacalone, V. Campisciano, C Calabrese, V. L. Parola, Z. Syrgiannis, M. Prato, M. Gruttadauria, ACS Nano 2016, 10, 4627-4636; d) B. Karimi, M. R. Marefat, M. Hasannia P. F. Akhavan, F. Mansouri, Z. Artelli, F. Mohammadi, H. Vali, ChemCatChem 2016, 8, 2508-2515.

[23] J. P. Knowles; A. Whiting, Org. Biomol. Chem. 2007, 5, 21-44

[24] The E-configuration of the double bond was assigned with NOESY NMR experiment.
[25] The yield was calculated by considering the use of two equivalents of 4bromo-acetophenone.

[26] a) M. Beller, T. H. Riermeier, Tetrahedron Lett. 1996, 37, 6535-6538; b) M. Beller, T. H. Riermeier, Eur. J. Inorg. Chem. 1998, 1998, 29-35.

[27] W. Z. Ostwald, Z. Phys. Chem., Stoechiom. Verwandtschaftsl. 1900, 34 495-503.

[28] Efforts to evaluate the catalytic behavior of that solution where hampered by the lack of techniques to separate the PdNPs@NG in operando due to the solubility of the support under catalytic conditions. Hot filtration test would be inconclusive as the hybrid nanogel would pass through filter membranes.

[29] a) J. Rebek, F. Gavina, J. Am. Chem. Soc. 1974, 96, 7112-7114; b) J. Rebek, D. Brown, S. Zimmerman, J. Am. Chem. Soc. 1975, 97, 454 455; J. Rebek, Tetrahedron 1979, 35, 723-731; d) J. P. Collman, K. M Kosydar, M. Bressan, W. Lamanna, T. Garrett J. Am. Chem. Soc. 1984 106, 2569-2579; e) J. M. Richardson, C. W. Jones, J. Catal. 2007, 251, 80-93; f) R. H. Crabtree, Chem. Rev. 2012, 112, 1536-1554.

[30] The polymeric scavengers can only sequester the palladium species that are leached out of the nanogel. Analogously, polymeric substrates can only undergo Mizoroki-Heck coupling if there are active palladium species outside the NG.

[31] a) S. Klingelhöfer, W. Heitz, A. Greiner, S. Oestreich, S. Förster, M. Antonietti, J. Am. Chem. Soc. 1997, 119, 10116-10120; b) J. M. Richardson, C. W. Jones, Adv. Synth. Catal. 2006, 348, 1207 - 1216; c) M. Weck, C. W. Jones, Inorg. Chem. 2007, 46, 1865-1875.

[32] These reactions were run at significant higher dilution than the previous tests to prevent artefacts, such as possible NG aggregation due to the interaction between polymer chains of the nanogel and P4VP.

[33] a) S. S. Pröckl, W. Kleist, M. A. Gruber, K. Köhler, Angew. Chem. Int Ed. 2004, 43, 1881-1882; b) C. C. Cassol, A. P. Umpierre, G. Machado, S. I. Wolke, J. Dupont, J. Am. Chem. Soc. 2005, 127, 3298-3299.

[34] J. G. de Vries, Dalton Trans. 2006, 421-429.

[35] X. André, M. Zhang, A. H. E. Müller, Macromol. Rapid. Commun. 2005 26, 558-563. 